\title{
2.6. Piaget visité par la didactique
}

\section{Gérard Vergnaud}

\section{Citer ce document / Cite this document :}

Vergnaud Gérard. 2.6. Piaget visité par la didactique. In: Intellectica. Revue de l'Association pour la Recherche Cognitive, n³3, 2001/2. Piaget et les sciences cognitives. pp. 107-123;

doi : https://doi.org/10.3406/intel.2001.1635

https://www.persee.fr/doc/intel_0769-4113_2001_num_33_2_1635

Fichier pdf généré le 24/02/2020 
Intellectica, 2001/2, 33

\title{
2.6 Piaget visité par la didactique
}

\author{
Gérard Vergnaud \\ Connaître ne consiste pas à copier le réel \\ mais à agir sur lui et à le transformer. \\ Jean Piaget (Biologie et Connaissance, p. 23)
}

Cette petite phrase annonce un principe fondamental de la théorie piagétienne de la connaissance comme activité du sujet. Piaget complète d'ailleurs ainsi cette proposition :

« de manière à le comprendre (le réel) en fonction des systèmes de transformation auxquels sont liées ces actions ».

Or Piaget n'a pas véritablement appliqué cette maxime à sa propre contribution scientifique de psychologue. En dépit de ses fonctions au Bureau International d'Education, il n'a pas cherché à agir sur les connaissances des enfants et à les transformer. Son intérêt était ailleurs, dans la clarification des liens entre évolution biologique et développement des connaissances, ce dont témoigne clairement son ouvrage «Biologie et Connaissance. ».

L'auteur du présent article est psychologue et didacticien. Ses contributions scientifiques portent principalement sur l'apprentissage des mathématiques et sur le développement des compétences tout au long de la vie. On ne sera donc pas étonné que les concepts et les thèses de Piaget soient réexaminées, réinterprétées, contredites et complétées par des considérations liées à ce champ scientifique, que Piaget avait négligé

\section{INTRODUCTION}

Piaget a fait plusieurs découvertes, comme celles concernant la conservation des quantités discrètes et continues, qui ne demandaient pas de technologie particulière, et que Platon ou Aristote auraient pu «théoriquement » faire. En fait cela leur était impossible parce qu'il ne fallait pas moins de toute l'histoire de la philosophie et des sciences pour que soient posés les problèmes auxquels la psychologie du vingtième siècle a commencé de répondre. L'œuvre de Piaget est à l'évidence une pièce maîtresse de cette psychologie du vingtième siècle, avec celle de Freud et celle de Vygotski.

Il est arrivé à Piaget une aventure peu commune : une reconnaissance internationale rapide, un immense flot de commentateurs, d'épigones et de contradicteurs, inégalement en mesure de comprendre les résultats et les interprétations du maître, un certain oubli voire une certaine condescendance à l'égard de l'œuvre pendant toute une période, le regain occasionné par la célébration du centenaire; sans compter les courants de recherche 
passés totalement à côté du message de Piaget, en raison d'hypothèques behavioristes et positivistes, de principes méthodologiques trop étroitement expérimentalistes, ou encore de visions excessivement réductionnistes. Comme toute science, la psychologie est réduction, mais toutes les réductions ne se valent pas. Il en est qui réduisent leur propos de telle sorte qu'elles laissent de côté des objets essentiels de la psychologie, notamment les processus de représentation consciente et inconsciente, et les compétences complexes qui s'expriment dans les activités quotidiennes, dans le travail et l'éducation. Piaget lui-même n'a pas échappé à des réductions discutables comme la tentative de réduction à la logique et à des stades généraux de développement, des opérations de pensée mathématiques, physiques ou morales. Mais en même temps, en prenant à bras le corps les problèmes de la représentation et de la connaissance, il a été pendant le vingtième siècle l'auteur le plus significatif de la psychologie cognitive antibehavioriste : c'est lui, davantage que tout autre psychologue, qui a su dénicher les connaissances sous-jacentes à l'activité de l'enfant en situation, et les fausses connaissances qu'immanquablement cette activité engendre. C'est un socle constitutif de la psychologie.

\section{QUELS CONCEPTS POUR PENSER L'ACTIVITE? QU'EST-CE QU'UN SCHEME?}

Piaget découvre le concept de schème dans les années vingt, probablement sous l'influence de Janet et de Revault d'Allonnes, et il lui donne un contenu empirique qui fait date, grâce à ses analyses de l'activité du bébé. Qu'il s'agisse de la coordination des mouvements des mains et des bras avec ceux du regard pour saisir et manier des objets matériels, qu'il s'agisse de l'utilisation de certains de ces objets comme instruments, qu'il s'agisse de la compréhension progressive de l'invariance des objets dans leurs déplacements et dans leurs disparitions derrière écran, Piaget place d'emblée ses analyses sur le plan de l'action sur et avec les objets matériels du réel, et sur le plan de la représentation. Bizarrement il utilise une terminologie («activité sensori-motrice, stade sensori-moteur ») qui reste prisonnière d'une caractérisation superficielle des phénomènes observés, et qui ne rend pas justice à ses propres analyses. Une expression comme " activité perceptivo-gestuelle " aurait été plus juste, tant il est vrai qu'il s'agit de sensations et d'actes moteurs organisés en perceptions et en gestes, pas seulement de sensorimotricité élémentaire.

Cette erreur «terminologique » n'est évidemment pas tragique; elle traduit néanmoins la difficulté rencontrée par les chercheurs, y compris les plus grands, pour trouver les mots les plus justes susceptibles de désigner leurs idées.

Assez rapidement, Piaget généralise le concept de schème aux raisonnements logiques et mathématiques. Un exemple est le schème dit de l'inclusion logique, qui consiste à reconnaître que le cardinal du tout est toujours plus grand que celui de la partie, à condition 
évidemment de ne pas confondre le tout avec le complément de la partie, ce qui justement fait problème pour les enfants jusqu'à un âge relativement tardif, entre 8 et 12 ans. On mentionne moins souvent dans la littérature les schèmes décrits par Piaget pour la construction de la représentation de l'espace, qui fournissent pourtant des cas exemplaires d'analyse de l'activité et des connaissances implicites : par exemple les gestes de vérification de l'alignement de plusieurs objets sont interprétés par Piaget comme un témoin et un critère de la conceptitualisation des propriétés affines de l'espace. Or ces gestes interviennent longtemps avant tout enseignement de la géométrie, ce qui l'autorise à parler de la géométrie «spontanée » de l'enfant. Evidemment Piaget sait bien qu'il y a une distance cognitive et épistémologique importante entre les connaissances implicites dans l'action et les connaissances explicites du mathématicien ou de l'élève apprenant les mathématiques, mais son mérite est justement d'avoir identifié, par l'analyse, des formes de conceptualisation qui ne se présentent pas comme des conceptualisations pour l'observateur tout venant.

On peut faire cependant plusieurs sortes de commentaires concernant l'analyse des schèmes par Piaget.

Premier commentaire: Revault d'Allonnes avait développé le concept de schème, plusieurs années avant Piaget, en l'inscrivant principalement dans une théorie de la reconnaissance et de la perception, il parle même de l'aperception, c'est-à-dire d'un processus de prise d'information rapide, et inévitablement réducteur. Son idée, déjà très intéressante, est que nous organisons l'information perçue en scènes schématiques et en silhouettes, ce dont témoignent non seulement les phénomènes psychologiques, mais aussi beaucoup de produits culturels comme les adages, les dictons, les enseignes. Revault d'Allonnes parle peu d'action et d'activité.

C'est parce qu'il étudie l'activité perceptivo-gestuelle des bébés que Piaget est amené à placer le concept de schème dans une théorie de l'action plus que de la perception. Il faut lui rendre hommage d'avoir fait cette rupture, car elle l'a conduit à considérer non pas des totalités principalement perceptives comme le faisaient les gestaltistes, mais des totalités dynamiques fonctionnelles, organisant le décours temporel de l'activité. Cette coupure n'a été faite ni par Revault d'Allonnes, ni par Bartlett, ce père anglo-saxon des schémas que certains psychologues continuent à confondre avec des schèmes; l'ouvrage de Bartlett est remarquable mais il laisse totalement de côté l'activité gestuelle. Or c'est dans les gestes qu'on trouve les meilleurs exemples de l'activité, les plus incontournables pour l'analyse de la pensée non verbale, et en même temps les modèles les plus suggestifs pour l'analyse de la pensée et du raisonnement. Le geste est pensée; le geste est un modèle suggestif pour l'analyse de l'activité de pensée. On peut même ajouter: la pensée est un geste.

Deuxième commentaire: Piaget indique d'emblée la valeur de généralisation du schème, dont il fait une sorte d'équivalent du 
concept au plan de l'action. "Les actions ne se succèdent pas au hasard mais se répètent et s'appliquent de façon semblable aux situations comparables. Plus précisément elles se reproduisent telles quelles si aux mêmes intérêts correspondent des situations analogues, mais se différencient ou se combinent de façon nouvelle si les besoins ou les situations changent. Nous appellerons schème d'action ce qui, dans une action, est ainsi transposable, généralisable ou différentiable, d'une situation à la suivante, autrement dit ce qu'il y a de commun aux diverses répétitions ou applications de la même action ». (Piaget, 1967a, p.16).

Mais il ne précise pas le concept de classe de situation, à laquelle justement s'adresse le schème dans sa généralité. Ce n'est pas une mince surprise, que de voir ainsi l'auteur d'un concept aussi essentiel que celui de schème manquer le concept dual qui lui donne sa portée et sa signification. Le couple conceptuel « schème-situation » est la clef de voûte de la psychologie cognitive et de la théorie de l'activité : pour cette raison simple que, la connaissance étant adaptation, ce sont les schèmes qui s'adaptent, et qu'ils s'adaptent à des situations. Piaget a beaucoup élaboré sur ce concept d'adaptation, inspiré qu'il était par la biologie; mais le plus souvent il en a formulé la dialectique dans des termes généraux comme interaction «sujet-objet » ou interaction « organisme-milieu » alors qu'il disposait de moyens théoriques et d'exemples pour en conduire des analyses plus fines, en analysant les relations entre les propriétés d'un schème et les propriétés de la classe de situations à laquelle il s'adresse. C'est la recherche en didactique (pour un exposé systématique voir Brousseau), qui a permis de sortir le concept de situation des oubliettes du sens commun, et d'en faire un concept scientifique et pratique, avec lequel il soit possible d'agir : notamment de produire des effets d'apprentissage et de prise de conscience, en manipulant certaines variables de situation. C'est un point essentiel pour l'éducation et le travail, que Piaget n'a pas su exploiter. C'est probablement mon expérience de chercheur en didactique des mathématiques qui m'a permis de donner du schème une définition plus précise que celle de Piaget.

\section{Définition 1 : Le schème est une organisation invariante de l'activité pour une classe définie de situations}

Le concept d'algorithme $\mathbf{m}$ 'a aidé à circonscrire cette définition, justement parce que les algorithmes sont des schèmes, bien que tous les schèmes ne soient pas des algorithmes évidemment. La parenté des schèmes avec les algorithmes est rendue particulièrement visible par l'analyse des erreurs : les élèves en prennent souvent à leur aise avec les règles des algorithmes qui leur sont enseignées et leur substituent des règles à eux, inspirées par les conceptions restrictives qu'ils ont de ce qu'est un nombre, une variable, une fonction, une opération, une écriture équivalente etc.... Par exemple la conception primitive, qui est la leur, que le nombre est une mesure, les empêche 
de traiter les nombres négatifs avec les mêmes règles que les nombres positifs: les mesures sont en effet toujours positives.

Evoquons rapidement trois exemples de schèmes dans le domaine des mathématiques :

- le schème du dénombrement consiste à associer à une collection d'éléments discrets, une suite de mots-nombres (un, deux, trois, quatre) et à identifier le cardinal de la collection par le dernier mot-nombre prononcé : le plus souvent en répétant ce mot-nombre ou en le soulignant toniquement (un, deux, trois, ... quatre). Ce schème, que les enfants développent à partir de trois ou quatre ans, n'est pas un schème purement verbal ou numérique : il suppose une correspondance biunivoque entre quatre catégories d'éléments distincts : les objets dénombrés, les gestes de la main et du bras, les gestes du regard, les gestes de la voix. La cardinalisation est une compétence distincte de la compétence à coordonner biunivoquement plusieurs registres de gestes. Certains enfants expriment l'une de ces deux compétences et pas l'autre. Le schème du dénombrement n'est pas un algorithme.

- l'algorithme de la division consiste, grâce au concours essentiel de la numération de position, en la division d'une partie du dividende par le diviseur (éventuellement par une partie du diviseur pour amorcer le processus), et à progresser à partir des unités d'ordre supérieur vers les unités d'ordre inférieur, y compris les décimales. Les élèves rencontrent beaucoup de difficultés dans l'apprentissage et la conduite de cette activité, et substituent fréquemment des schèmes idiosyncratiques à l'algorithme enseigné par le maître. L'algorithme de la division est un schème, mais il lui arrive fréquemment de se dégrader en schèmes non algorithmiques (Brun et al, 1994).

- La résolution d'un système d'équations algébriques repose sur un système de signifiants/signifiés lentement élaboré au cours de l'histoire (et d'une toute autre nature que le système de signifiants/signifiés de la numération que nous venons d'évoquer). La résolution se prête à des algorithmes savamment élaborés par les mathématiciens au cours de l'histoire. Les élèves de collège s'écartent souvent de ces algorithmes et leur substituent des schèmes personnels, éventuellement efficaces, éventuellement erronés.

Dans les trois cas que nous venons d'évoquer, l'activité consiste en une succession de prises d'information et d'actions; ces prises d'information sont à chaque fois partielles, et les actions sont locales, de même que les contrôles qui émaillent le déroulement de l'activité.

Dans les trois cas, il y a un but et des sous-buts. Dans les trois cas l'organisation de l'activité repose sur des conceptualisations peu 
explicitables par les élèves, ainsi que sur des inférences en situation (anticipations, coordinations diachroniques et synchroniques...).

La définition du schème comme organisation invariante, me conduit à préciser que c'est l'organisation qui est invariante, non pas l'activité, ni la conduite : le schème n'est pas un stéréotype, l'algorithme non plus. Un schème engendre une diversité de conduites et d'activités selon les caractéristiques particulières des situations rencontrées. Cette fonction d'adaptation aux situations conduit à identifier dans le schème des règles SI... ALORS... qui relient les actions à des conditions et circonstances.

Le caractère général du schème, bien vu par Piaget, appelle donc une analyse en termes de règles conditionnelles, ratée par Piaget.

Troisième commentaire: Piaget a eu le mérite de repérer le caractère fondamentalement cognitif du schème, et bizarrement il n'a pas fait pour autant la liaison complète avec cet autre concept piagetien qu'est celui d'invariant opératoire. Dans ses écrits le concept d'invariant reste un peu prisonnier du contexte des situations de conservation dans lesquelles Piaget l'a illustré magistralement, tandis que celui de schème est davantage lié au décours temporel de l'activité.

C'est aux expériences piagétiennes relatives à la conservation des quantités discrètes et continues que je faisais allusion, au début de cet article, lorsque j'évoquais la possibilité, toute théorique évidemment, que Platon et Aristote auraient pu les faire. Ce que nous montre Piaget vingt-cinq siècles plus tard, c'est que l'évidence n'est pas une donnée de l'expérience perceptive mais résulte d'une construction, et suppose la coordination entre elles de plusieurs opérations de pensée et de plusieurs sortes d'informations.

L'équivalence numérique de la suite des coquetiers et de la suite des œufs, ou encore la conservation de la quantité de pâte à modeler, de sa masse et de son volume lorsqu'on la déforme, ne résultent pas d'une lecture pure et simple de l'expérience mais sont posées par l'enfant, à partir de certains moments du développement et de l'expérience, comme des moyens de comprendre de manière cohérente, un système de relations et de transformations se prêtant à des critères perceptifs contradictoires : (écartement, dépassement, déformation, morcellement...).

La définition des invariants par les mathématiciens à la fin du $19^{\text {ème }}$ siècle (Klein par exemple) correspond bien à cette idée, reprise par Piaget : «ce qui se conserve sous certaines transformations ». Mais il faut aller au-delà et considérer que c'est le fond le plus général de la pensée qui est concerné par cette idée de «ce qui est le même sous le différent ». Cela concerne aussi bien les variations entre objets d'une même classe, entre les situations d'une même classe, entre les différentes valeurs d'un même descripteur, entre les différents exemples illustrant une même relation à deux ou plusieurs termes, entre les différentes relations partageant des propriétés 
communes, comme c'est le cas par exemple des relations d'ordre, des relations d'équivalence, des lois de composition, etc.

Les concepts d'objet, de prédicat et de proposition relèvent tous les trois de cette même idée d'invariant :

- Tel objet reste lui-même bien que son apparence perceptive ait changé (sous l'effet d'une rotation, d'un éloignement, d'une disparition partielle), ou qu'il ait disparu et ne soit plus directement accessible à la perception.

- Telle propriété ou telle relation est pertinente pour sélectionner l'information utile à l'activité, dans une variété de situations.

- Telle proposition est vraie, ou tenue pour vraie dans l'action, pour une certaine classe de situations.

On doit même aller plus loin et considérer que la reconnaissance de prédicats et de propositions invariantes permet de construire des objets nouveaux, non directement accessibles à la perception. Ces objets résultent d'une construction imaginaire, dont témoigne en premier lieu, chez l'enfant, l'évocation d'objets réels en leur absence, et en second lieu la construction scientifique ou artistique d'objets de pensée nouveaux, par abstraction, métaphore ou autre processus créatif, et ne correspondant directement à aucun percept.

Il est important de comprendre, comme nous l'enseignent les logiciens, que les propositions sont susceptibles de vérité ou de fausseté, alors que les objets et les prédicats (concepts) ne sont ni vrais ni faux mais seulement pertinents ou non pertinents pour la prise d'information nécessaire à l'action. Il faut remarquer en même temps qu'il n'y a pas de proposition sans concepts, et pas de concept sans propositions. Concepts et propositions sont donc dans une relation dialectique, mais il ne faut pas les confondre pour autant. Objets et prédicats sont eux-mêmes dans une position toute relative : ce qui est prédicat aujourd'hui peut devenir objet demain pour le même enfant, et se voir attribuer de nouveaux prédicats. Par exemple le prédicat «symétrique » donne naissance à l'objet « symétrie », qui désigne une transformation dans le plan ou dans l'espace, el qui, à son tour, est susceptible de prédication: «la symétrie conserve les distances $»$.

Piaget n'a pas saisi pleinement la puissance du concept d'invariant opératoire, et ne l'a pas non plus relié au concept de schème autant qu'il aurait été utile de le faire.

C'est ce double problème du caractère conditionnel de l'activité et de sa liaison avec la conceptualisation du réel que j'ai, pour ma part, essayé de résoudre. La définition analytique du schème cidessous résulte directement de cette préoccupation. 


\section{Définition 2 : Un schème comporte nécessairement quatre} composantes :

- un ou plusieurs buts, avec leur cortège de sous-buts et d'anticipations,

- des règles d'action, de prise d'information et de contrôle,

- des invariants opératoires (concepts-en-acte et théorèmesen-acte) permettant à la fois la prise et le traitement de l'information pertinente,

- des possibilités d'inférence.

Ces quatre composantes sont absolument essentielles. Intentionnalité, générativité, conceptualisation sont obligatoirement au rendez-vous, fussent-elles totalement implicites voire inconscientes. Quant aux inférences, elles sont tout autant nécessaires : en effet, comme le schème n'est pas un stéréotype, il appelle toujours des «calculs inférentiels» en situation. Ce que le scientifique fait le plus explicitement et le plus complètement possible en raisonnant, la pensée en acte le fait spontanément, de manière implicite et lacunaire le plus souvent, mais avec un certain succès.

Dans les travaux issus de la tradition Newell et Simon et concernant le réglage de l'activité, on ne trouve ni le rôle de la conceptualisation, ni celui de la prise d'information. C'est une grave lacune, que la métaphore informatique n'a toujours pas surmontée.

\section{LOGIQUE OU CONCEPTUALISATION : QU'EST-CE QU'UN CHAMP CONCEPTUEL ?}

La tentation de considérer le jugement et le raisonnement comme relevant de la logique n'a pas épargné Piaget. C'est probablement la tradition philosophique depuis Aristote qui l'a entraîné dans cette voie, également l'effort quasi surhumain des logiciens depuis le $19^{\text {eme }}$ siècle pour réduire les connaissances mathématiques à des systèmes axiomatiques complets et économiques, peut-être aussi la recherche d'une théorie générale de la pensée, dont on n'apercevait pas au milieu du $20^{\text {enre }}$ siècle, qu'elle ne pouvait pas relever seulement de la logique des propositions, ou de la logique des prédicats.

Gödel avait déjà produit sa fameuse démonstration que la logique ne permet pas de décider à elle seule de la non contradiction de l'arithmétique. Mais la leçon n'en avait pas été tirée qu'il fallait considérer la logique comme un champ conceptuel parmi d'autres, permettant peut-être d'avoir un regard critique sur les énoncés des autres champs conceptuels, mais ne permettant ni de les engendrer, ni de les coiffer. On ne peut pas s'appuyer sur la logique pour comprendre les principes de la géométrie, de la mécanique, de la chimie, de la thermodynamique, de la biologie ou de l'histoire. 
Il nous faut donc faire une petite révolution épistémologique et placer au centre des processus cognitifs, non point la logique mais la conceptualisation : cela signifie qu'il faut considérer au plus près la diversité des compétences et des formes que peut prendre la conceptualisation. C'est du moins la position d'un chercheur en didactique qui s'intéresse aux problèmes d'apprentissage et d'enseignement d'une variété de disciplines et de sous-disciplines, ainsi que d'une variété de professions et de pratiques professionnelles. C'est également la position des historiens et des épistémologues des sciences et des techniques aujourd'hui.

Piaget s'est donc engagé dans une voie peu féconde lorsqu'il a cherché à formaliser dans des systèmes logiques, ou voisins de la logique comme la structure de groupement, les opérations de pensée de l'enfant au stade des opérations dites « concrètes » et au stade des opérations dites «formelles ». Le concept de groupement ne permet pas de saisir convenablement la variété des compétences nouvelles des enfants entre 7 et 10 ans concernant les conservations, les sériations, ou la représentation de l'espace alors étudiées par Piaget et ses collaborateurs. Le groupe dit INRC des quatre opérations propositionnelles (identique, inverse, réciproque et corrélative) ne caractérise pas non plus les opérations conceptuelles qui sont accessibles aux enfants de 12 ans et plus. La combinatoire et le raisonnement hypothético-déductif non plus, puisqu'on peut les observer chez des enfants beaucoup plus jeunes.

La logique apparaît aujourd'hui comme un biais réductionniste dans l'ouvre de Piaget. Un exemple me permettra de justifier la sévérité de cette appréciation.

La compréhension de la proportionnalité est un domaine auquel s'est intéressé Piaget. A sa suite ou de manière indépendante, de nombreux psychologues se sont engagés dans l'étude des raisonnements et des biais de raisonnement concernant la multiplication, la division, la recherche d'une quatrième proportionnelle ou l'équilibre de la balance. Le bilan de ces recherches fait apparaître aujourd'hui qu'on ne peut pas faire l'économie des concepts de fonction linéaire, de fonction bilinéaire, et de leurs propriétés d'isomorphisme, pour caractériser les différents raisonnements et procédures utilisés par les enfants ou les adultes; on ne peut davantage se passer des concepts de fonction directe et inverse, de coefficient de proportionnalité, de dépendance et d'indépendance.

Dans le domaine de la proportionnalité il existe de nombreux raisonnements possibles. Certains sont plus aisément compris et utilisés par les enfants de 9 ou 10 ans : ce sont ceux qui reposent sur les propriétés d'isomorphisme de la fonction linéaire:

$$
\begin{aligned}
& f\left(x+x^{\prime}\right)=f(x)+f\left(x^{\prime}\right) \\
& f(k x)=k f(x) \\
& f\left(k x+k^{\prime} x^{\prime}\right)=k f(x)+k^{\prime} f\left(x^{\prime}\right)
\end{aligned}
$$


D'autres raisonnements sont plus délicats, même pour des élèves de 15 ans, comme celui qui sous-tend la technique du produit en croix Une perspective développementale est donc bienvenue. Mais ce sont souvent les mêmes raisonnements qui sont compris plus aisément par les enfants ou les adolescents, et par les adultes, notamment par ceux qui n'ont pas eu de grandes réussites en mathématiques.

En outre les interprétations de Piaget sont très discutables, en tout cas très insuffisantes. Par exemple la compréhension de l'équilibre de la balance, ne relève pas fondamentalement d'un raisonnement s'appuyant sur le groupe INRC des quatre transformations propositionnelles, sauf par une analogie relativement superficielle. On a à faire à des grandeurs variables et à leurs relations. Le moment des forces sur chacun des bras est une fonction bilinéaire de la masse accrochée et de la distance entre le couteau et le point d'accrochage. L'indépendance des deux variables est essentielle, et suppose des théorèmes implicites du type : «c'est proportionnel à la distance quand la masse est tenue constante, et proportionnel à la masse quand la distance est tenue constante $\gg$.

La proportion inverse entre poids et distance n'est vraie que lorsqu'on ne change rien sur l'autre bras du fléau : on tient alors constant le produit masse $\mathrm{x}$ distance. Pour comprendre l'équilibre de la balance, le concept de moment (ou son équivalent implicite) est donc essentiel, ainsi que celui de bilinéarité.

Le groupe INRC ne traduit presque rien du réseau des relations entre variables quantitatives. D'une manière plus générale d'ailleurs, dès que des grandeurs sont en jeu, l'approche logique apparaît ridiculement insuffisante. Cette insuffisance est déjà visible pour le concept de nombre, pour lequel Piaget sous-estime le critère de l'addition, alors que c'est justement l'addition qui distingue radicalement les relations numériques des relations d'ordre et d'équivalence; le nombre n'est pas la synthèse opératoire des classes et des relations d'ordre, même si classe et ordre sont des conditions nécessaires du concept de nombre. Le formalisme de la théorie de la mesure

Card $(A \mathrm{U} B)=$ ard $(\mathrm{A})+\operatorname{card}(\mathrm{B})$ pourvu que $\mathrm{A}$ et $\mathrm{B}$ soient disjoints,

caractérise mieux que tout formalisme logique la conquête cognitive que représente l'acquisition du concept de nombre. La découverte de la non-conservation des quantités discrètes chez les jeunes enfants, découverte remarquable bien entendu, a probablement empêché Piaget de saisir plus complètement les progrès de la quantification. sévère.

Ecrivant ce que je viens d'écrire, j'ai conscience d'être trop

En effet, dans le même temps où il tombait dans un certain réductionnisme logique, Piaget conduisait des recherches qui montraient son intérêt pour des opérations de pensée qui ne 
relevaient pas de la logique mais de la conceptualisation de l'espace, $\mathrm{du}$ mouvement, du hasard. Ce Piaget-là reste d'un grand intérêt aujourd'hui, et montre que les conceptualisations spécifiques des différents domaines de connaissance ne lui étaient pas étrangères. Simplement il ne s'est pas engagé dans leur étude aussi complètement que dans la perspective logiciste, qu'il a cru plus puissante qu'elle ne l'était en réalité. En outre, en s'intéressant au développement de l'intelligence et de la pensée, et pas à l'éducation, Piaget n'a pas vu que les problèmes d'apprentissage à l'école pouvaient apporter à son épistémologue génétique, une abondante moisson de phénomènes. Encore fallait-il pour cela qu'il reconnaisse dans les processus de conceptualisation spécifiques des disciplines et des sous-disciplines scolaires, des processus dignes d'intérêt pour la théorie générale qu'il voulait élaborer. Ce n'était pas le cas.

La didactique ne pouvait évidemment pas se contenter d'une approche piagétienne pour décrire et analyser les processus d'apprentissage et les méthodes d'enseignement. Elle s'est donc inspirée aussi de l'épistémologie des disciplines et de leur histoire. Il était plus fécond pour les didacticiens des mathématiques de comprendre les difficultés des concepts de nombre, de figure, de repère, de variable, de fonction, à travers les transformations qu'ont subies ces concepts au cours de l'histoire, et qu'ils subissent au cours des apprentissages scolaires, que de chercher à utiliser les formalisations de Piaget concernant les stades. De même pour les didacticiens de la physique ou de la biologie.

Pourtant, une approche développementale est essentielle pour la recherche en didactique, et c'est une grande leçon pour les chercheurs que de constater que Piaget a été une référence incontournable dans les milieux de l'éducation alors que son entreprise scientifique ne porte pas directement sur l'éducation.

Le cadre théorique des «champs conceptuels » est né de cette préoccupation d'avoir les moyens de saisir les filiations et les ruptures au cours des apprentissages scolaires et professionnels. Si on ne peut pas s'en tenir à des stades généraux de pensée totalement ordonnés, mais qu'il faut au contraire saisir le développement des compétences et des conceptualisations dans leur variété et leurs différences, on est conduit à plusieurs prises de position, que je voudrais maintenant résumer.

- Les situations que les enfants sont progressivement en mesure de maîtriser forment un ensemble partiellement ordonné, jamais totalement ordonné, sauf localement.

- Les compétences résultent des formes d'organisation de l'activité mises en œuvre, c'est-à-dire des schèmes : ceux-ci sont à la fois sous le contrôle de l'institution chargée de les transmettre (famille, école, communauté de travail) et sous le contrôle du sujet individuel qui apprend.

- Les situations sont à la fois la source et le critère de la connaissance. C'est en situation qu'on apprend; une 
connaissance qui n'est pas opératoire n'est pas véritablement une connaissance.

- La collaboration et la communication avec autrui, pair ou adulte plus expert, est essentielle dans la formation des schèmes et des conceptualisations nécessaires à la réussite.

- Le langage transforme les concepts-en-acte et les théorèmes-en-acte en concepts et en théorèmes susceptibles d'être communiqués et débattus, quant à leur pertinence et leur vérité.

- La mise en mots est une compétence difficile à acquérir, y compris pour les professionnels et les scientifiques de haut niveau. Un traité scientifiquc ou technique est la partie visible d'un iceberg dont la majeure partie repose sur l'action et l'expérience.

Un champ conceptuel est par définition un ensemble de situations et un ensemble de concepts. Ce sont les situations qui donnent leur sens aux concepts, par le biais de l'activité du sujet apprenant; ce sont les concepts-en-acte et les théorèmes-enacte contenus dans les schèmes qui permettent de traiter ces situations. Un bon exemple est celui des structures additives. Le concept de nombre n'émerge pas avec toutes ces propriétés au cours de l'apprentissage-développement de l'enfant, mais seulement avec certaines propriétés succintes qui permettent à l'enfant de comprendre et de traiter un petit nombre de classes de situations : augmentation (connue) d'une petite collection d'éléments discrets (connue également), réunion de deux parties (connues) en un tout. Ces situations prototypiques ne sont pas des modèles suffisants pour traiter d'autres classes de situations, pourtant voisines des deux premières, notamment la recherche d'un état initial lorsqu'on connaît l'état final et la diminution qu'elle a subie, également les différents cas de soustraction, qui sont très nombreux. Lorsque des transformations de signes différents sont composées et décomposées, les opérations d'addition et de soustraction deviennent délicaates, au point qu'une majorité d'élèves de 15 ans sont tenus en échec par un problème qui ne demande qu'une addition, laquelle représente en fait la différence entre deux transformations de signe contraire. Les opérations de pensée nécessaires pour décider de l'opération à faire supposent des conceptualisations relativement complexes, qui sont représentables par des calculs dans l'ensemble des nombres relatifs, mais qui ne sont pas accessibles à tous les élèves, même lorsqu'ils ont appris les nombres relatifs.

Un autre exemple de champ conceptuel est celui de la reproduction végétale, pour lequel le système des concepts quotidiens (fleur, fruit, graine) est à la fois un point d'appui et un obstacle pour les conceptualisations académiques transmises par l'école.

Les concepts forment système, mais le système ainsi formé ne se construit pas d'un seul coup. Il se développe au cours de l'expérience, en fonction des situations rencontrées et des 
provocations organisées par les enseignants, la famille, les formateurs. Il est donc le produit de l'activité du sujet, face aux événements contingents de son histoire personnelle et professionnelle. Au cours de ce processus, on observe à la fois des filiations et des ruptures. Les connaissances antérieures sont à la fois des points d'appui, et des obstacles possibles pour les connaissances plus évoluées.

Les connaissances formulées en mots, énoncés et textes, reposent sur un ensemble considérable de rencontres avec des situations d'action et d'échange avec autrui. La logique joue un rôle relativement faible dans ce processus.

\section{ACTION, OPERATION, PERCEPTION, ABSTRACTION, LANGAGE : TOUT A-T-IL ETE DIT?}

Piaget a eu beaucoup d'idées. Qu'elles n'aient pas toutes été aussi heureuses, les unes que les autres n'est pas pour surprendre. En mettant l'accent sur les opérations, et leur structuration dans des «structures d'ensemble» tels que groupements et groupes, Piaget marquait ses distances à la fois par rapport à la perception et au poids de la perception dans les théories de la pensée, et par rapport à l'action simple, celle qui permet d'agir sur les objets matériels et sociaux. Etre actif pour Piaget, cela ne se résume pas dans l'action matérielle sur des objets manipulables (comme ceux conçus intentionnellement pour l'apprentissage des bébés et des enfants), c'est aussi et surtout opérer en pensée sur les propriétés des objets et sur leurs relations : par des négations, des inversions, des annulations, des combinaisons... En d'autres termes c'est voyager dans un système coordonné d'opérations de pensée, qui s'appuient certes sur des actions matérielles, pour certaines d'entre elles, mais qui ne sont pas toutes dépendantes des actions matérielles, tant s'en faut, puisque le monde des possibles devient à l'adolescence, selon Piaget, l'ensemble de référence dont le monde réel n'est qu'un cas particulier.

Il faut porter au crédit de Piaget cette audace théorique. Mais faut-il pour autant opposer des processus figuratifs et des processus opératifs, qui sont à l'évidence inséparables dans l'activité, puisqu'il $\mathrm{n}^{\prime} \mathrm{y}$ a pas d'action sans prise d'information, et que la structure des perceptions et des évocations est un déterminant essentiel de l'organisation de l'action. D'ailleurs la perception implique des opérations de pensée importantes.

Si la perception met en jeu des opérations de pensée plus complexes qu'il n'y paraît, c'est parce que sa fonction est l'identification des situations, des objets, de leurs propriétés et relations, ainsi que des processus de transformation auxquels ils se prêtent. En d'autres termes la perception est conceptualisation, et elle n'est ni donnée toute faite par l'appareil génétique, ni simple lecture passive des situations vécues. Bartlett cite cet exemple éloquent de trois personnages se promenant dans la montagne; l'un est géologue, l'autre spécialiste de biologie végétale, le troisième peintre. Ils ne 
perçoivent pas la même chose, nous dit Bartlett : ni leur intention, ni leurs catégories de pensée ne les conduisent à prélever les mêmes informations. La perception est activité. C'est d'ailleurs ce que montre Piaget lui-même dans de nombreuses recherches sur la perception. Ainsi l'aspect opératoire de la perception doit-il être reconnu comme tel.

Si l'on approfondit un peu cette idée, à la lumière du concept de schème défini plus haut, il apparaît en effet que la prise d'information et le contrôle sont des conditions essentielles de l'efficacité de l'action.

Dans l'exemple du schème du dénombrement, les enfants qui échouent durablement à assurer la correspondance biunivoque entre les objets et la suite des mots-nombres, comme c'est le cas de certains enfants cérébro-lésés, sont justement ceux qui ne parviennent pas à organiser la séquence des regards nécessaires.

L'activité perceptive et la prise d'information font donc partie de l'activité opératoire. C'est même une compétence critique dans un sport collectif comme le football, compte-tenu de la rapidité du jeu. Dans le traitement d'un système d'équations algébriques, on observe également, en étudiant les erreurs des élèves de collège, que beaucoup d'erreurs tiennent à une prise d'information lacunaire ou erronée des informations contenues dans les lignes qui précèdent la ligne qui fait l'objet de l'activité actuelle. La conceptualisation est essentielle dans le choix de l'information pertinente, mais en retour le choix de l'information pertinente est essentiel dans l'activité conceptuelle.

On peut se demander pourquoi Piaget revient régulièrement dans certains ouvrages, sur l'opposition perceptif/opératif. Certes les deux ne sont pas une seule et même chose et Piaget a le souci des analyses fines. Mais il me semble que cette dualité n'est pas innocente et alimente certaines des thèses importantes de Piaget contre l'empirisme : la distinction entre abstraction simple et abstraction réfléchissante, ou la différence entre la pensée dite «logicomathématique » et la pensée physique.

Piaget a été impressionné par le fait que les mathématiques fournissent, sur la base, estime-t-il, du seul raisonnement formel, des théories qui se révèlent adéquates, après coup et de manière quasi miraculeuse, pour modéliser les phénomènes empiriques de la physique. On peut même considérer que cette distinction entre connaissance logiquo-mathématique et connaissance physique est dans certains ouvrages de Piaget, comme «l'introduction à l'épistémologie génétique ", plus décisive encore que la référence à la biologie.

Il y a donc lieu de s'interroger sur cette conception de Piaget des rapports entre connaissance du monde physique et connaissance mathématique. Elle est marquée au coin de l'idéalisme philosophique, et ce n'est pas un mince paradoxe que Piaget adopte une telle position alors qu'il avait lui-même étudié avec un certain 
détail le rôle de l'activité matérielle de l'enfant dans la conceptualisation de l'espace. C'est donc en remettant au centre de la réflexion et de la recherche en psychologie les schèmes d'activité matérielle sur les figures, les positions et les transformations spatiales, ainsi que ceux concernant les quantités et les grandeurs mesurables qu'on peut rétablir sans les opposer les relations entre connaissances mathématiques et connaissances physiques. On retrouve alors la dimension pragmatique des mathématiques, comme ensemble structuré de domaines d'activité et de conceptualisation. Par exemple on voit mal pourquoi on opposerait la mécanique comme théorie du mouvement, à la géométrie comme théorie du déplacement. Pourquoi la première relèverait-elle de l'abstraction simple et la seconde de l'abstraction réfléchissante ?

Il faut donc s'intéresser aux processus d'abstraction pour dénicher certains glissements de la théorie piagétienne. Pourtant c'est justement en distinguant plusieurs processus d'abstraction que Piaget a pu avancer des idées suggestives sur la conscience et la prise de conscience, idées qui rejoignent à bien des égards celles de Vygotski. Vygotski évoque à plusieurs reprises dans son ouvrage «Pensée et Langage » l'effet en retour de l'apprentissage de la langue écrite sur celui de la langue orale, de l'apprentissage d'une langue étrangère sur celui de la langue maternelle, ou encore de l'apprentissage de l'algèbre sur celui de l'arithmétique.

Plus généralement il distingue deux niveaux de conscience, celui qui permet de faire, et qui conditionne la réussite (conscience-avant), et celui qui permet de revenir sur les raisons de la réussite ou de l'échec, et qui conditionne la stabilisation et l'explicitation des connaissances (conscience-après). Si l'on rapproche cette analyse de celle de Piaget, on peut dire que la «conscience-avant » est nécessaire pour réussir, la « conscience-après » pour comprendre et débattre avec autrui des conditions de la réussite.

Ainsi, avec la distinction entre «Réussir et Comprendre », et avec l'idée que l'abstraction réfléchissante porte sur les opérations du sujet connaissant, Piaget nous livre une thèse profonde et utile pour l'éducation et rejoint de manière spectaculaire le point de vue de Vygotski. Mais alors pourquoi les connaissances de physique, de biologie, de morale ou de linguistique seraient-elles privées de ce processus d'abstraction réfléchissante?

En fait, ce n'est pas tant la distinction entre abstraction simple et abstraction réfléchissante qui fait problème, mais l'attribution de la première à certaines sciences considérées comme empiriques et l'attribution privilégiée de la seconde aux mathématiques, considérées comme non-empiriques.

Pour la didactique des mathématiques aujourd'hui, il est bien difficile de se priver de l'idée que les mathématiques sont en leur début une connaissance pragmatique, comme les autres sciences. Les mathématiques sont alors, pour les élèves une connaissance issue de l'expérience des situations dans lesquelles on a besoin de mesurer, de 
combiner et décombiner des mesures, d'identifier des figures et d'analyser leurs propriétés, de repérer des positions et des changements de position, de comprendre, dans les transformations géométriques et numériques, ce qui est modifié et ce qui est conservé. C'est seulement dans une phase relativement tardive de leur développement que les structures mathématiques deviennent des systèmes axiomatisables et formalisables. Il ne faut donc pas confondre l'après et l'avant.

Il se trouve cependant que les champs conceptuels qui permettent de circonscrire et d'analyser les processus de développement cognitif et les apprentissages scolaires en mathématiques, sont assez rapidement assujettis à des systèmes de relations nécessaires entre elles, alors que les champs conceptuels de la physique laissent une marge d'incertitude plus grande entre les relations qui les caractérisent, en raison des variables cachées, et donc hypothétiques, et des limites des processus d'approximation.

\section{QUELLE PLACE POUR LE LANGAGE ET LE SYMBOLISME?}

Piaget a eu le mérite de débusquer les connaissances implicites dans l'action. Ce faisant, il s'est probablement désintéressé un peu trop du rôle du langage et du symbolisme. Pourtant son idée d'opération formelle aurait dû le conduire à regarder de près le rapport de ces opérations avec les manipulations symboliques, notamment en mathématiques. Il ne l'a guère fait et sa propre passion pour les symbolismes formels (son traité de logique par exemple) ne l'a pas conduit à rechercher chez les adolescents les traces d'une rupture qualitative dans l'usage des symbolismes.

C'est donc un chantier qui reste très ouvert, tant il est vrai que la mise en mots et en symboles occupe une large place de l'activité humaine aujourd'hui, dans l'éducation et dans le travail.

Les schèmes langagiers, discursifs et communicatifs ont commencé d'être étudiés depuis une vingtaine d'années, surtout pour les conversations courantes, pas assez pour les conversations professionnelles, ni pour les échanges en classe. Ils permettent néanmoins de mieux comprendre les rapports entre connaissancesen-acte et connaissances explicites.

Qu'apporte l'invariance du symbolisme aux invariants opératoires ? Quel effet en retour le symbolisme utilisé par les scientifiques et les enseignants a-t-il sur la conceptualisation qui intervient dans l'action. On voit bien que c'est encore vers le concept de schème et vers le couple situation-schème, qu'on se retourne à nouveau pour espérer avancer.

Les schèmes de mise en mots sont associés à des situations d'énonciation et de débat, sur lesquelles peu d'analyses scientifiques ont encore été conduites. Mais on peut cependant considérer que la boucle est bouclée. La forme opératoire de la connaissance, d'abord recherchée dans l'action qui réussit, se distingue clairement de la forme prédicative de la connaissance, qui consiste en énoncés et en 
textes. Mais dès qu'on prend conscience d'une part, du poids de la communication et du caractère intersubjectif de la connaissance construite dans l'éducation et le travail, $d$ 'autre part de la fonction de représentation des formes langagières et symboliques, on est conduit à reconnaître la valeur opératoire de la forme prédicative de la connaissance.

Non seulement on agit sur autrui en lui parlant, ce que les linguistes pragmatistes affirment depuis plusieurs décennies déjà, mais on agit sur soi-même en modifiant le statut de ses propres connaissances : une connaissance mise en mots et en symboles n'est plus la même connaissance. 